\title{
The effect of emotional valence and age of faces on adults and children's inattentional blindness
}

\author{
Xiuying Wei ${ }^{1,2} \cdot$ Hui Zhang ${ }^{1} \cdot$ Jiangbo $\mathrm{Hu}^{3} \cdot$ Jinya $\mathrm{Xu}^{4}$. Jiale Wang ${ }^{4}$
}

Accepted: 24 November 2020 / Published online: 6 January 2021

(C) The Psychonomic Society, Inc. 2021

\begin{abstract}
Inattentional blindness (IB) refers to the nature of an individual being unaware of an unexpected stimulus when focusing on an attentional task. Investigation into IB provides an innovative approach for the research of attentional bias that is connected with an individual's immediate attentional capture. This study explored the effect of emotional valence and age of faces on the IB rates of children and adults. We employed a between-subject experimental design with a 2 (age of participants: child, adult) $\times 2$ (age of face: child face, adult face) $\times 2$ (emotional valence: positive, negative) method and used the classic IB task of cross judgment. The participants included 79 adults ( $54.63 \pm 8.192$ years old) and 78 children (10.62 \pm 2.32 years old). The results showed that: (1) The IB rates of the two groups differed significantly. Children performed better than adults in detecting the faces appearing unexpectedly. (2) The effect of emotional valence only emerged on adult participants' IB. Adults were significantly more likely to detect the positive-emotional faces than the negative-emotional ones. (3) The age of a face was not a significant factor to predict participants' IB. Neither children nor adults showed a preference for the faces of a specific age (e.g., their own age). These findings revealed the distinct characteristics of attentional capture of children and adults when confronting unexpected facial stimuli.
\end{abstract}

Keywords Inattentional blindness $\cdot$ Face age $\cdot$ Emotional valence $\cdot$ Children $\cdot$ Adults

\section{Introduction}

Inattentional blindness (IB) is an individual's instinct to reduce interruption from unexpected stimuli while concentrating on a task that needs close attention (Mack \& Rock, 1998). For example, when a person is engaged in conversation with a friend on a mobile phone, this person is unlikely to notice another friend who is smiling to him/her on the street though the friend is technically within his/her view. In recent years, IB has attracted researchers' interest in the field of attentional

Hui Zhang

zhanghui1987@zjnu.cn; 707183818@qq.com

1 Hangzhou College of Preschool Teacher Education, Zhejiang Normal University, 125 Wener Road, West Lake District, Hangzhou, China

2 Zi Jinghua School, Hangzhou, China

3 Macquarie School of Education, Faculty of Arts, Macquarie University, Sydney, NSW, Australia

4 Early Childhood Education Group in Zhejiang Normal University, Hangzhou, China process. IB provides a new and effective angle for the investigation of individuals' attentional bias under an inattentional condition in which the stimuli appear unexpectedly (Eastwood et al., 2003; Lee \& Telch, 2008).

Previous studies confirm that IB is associated with the types of stimuli. Stimuli containing abundant social information such as emotions, gender, or ethnic background would be more likely to be captured and processed by an individual's brain even when the person's attention is focused on another issue (Huang et al., 2012; Mack et al., 2002). Compared to neutral objects (e.g., simple geometric figures), self-relevant stimuli, such as participants' own names or warning words, are also more captivating to people's awareness when they appear unexpectedly. Therefore, faces revealing extensive social information including age, gender, race, and emotional information can be more likely to attract an individual's attention under an inattentional condition. This study aimed to explore the effect of a variety of faces of different ages and emotional valence on the IB of a group of school-aged children and mature middle-aged adults. This study will enhance our understanding of IB in these two age groups that are in totally different life stages, which may give an insight into the human developmental trajectory of the attentional process. 


\section{Research relating to the attentional process with face detection}

It is well established in the literature that the image of faces can capture people's attention better than other objects even when they appear unexpectedly. In an IB study, Devue et al. (2009) presented eight unexpected stimuli including four oval-shaped objects (a lemon, a strawberry, a potato, and a pear) and four faces (two males and two females) to a group of adults who were focused on a cross-judgment task (judging which arm of a cross is longer). The researchers found that the detection rate of the face pictures was $58 \%$, which is significantly higher than the detection rate of oval-shaped pictures at only 4\%. This result is in accordance with another study undertaken earlier by Mack and Rock (1998) who selected a set of cartoon faces as unexpected stimuli in the same attentional task, and the results showed that all of the cartoon faces (happy face, sad face, scrambled face) had higher detection rates than circle figures.

Yet other studies investigated the effect of emotional valence of faces on IB (Ge \& Jing, 2015; Wang et al., in press). Ge and Jing (2015) found that college students showed more sensitivity in detecting sad faces than happy faces that were presented in both real and abstract images when the students were instructed to count the number of four white objects crashing at the edge of a computer screen. However, in a similar experiment, when the participants were replaced with young children, the results showed that preschoolers' detection rates of positive-emotional faces were significantly higher than those of negative-emotional faces while they were busy with a cross-judgment task (Wang et al., in press). Interestingly, the positive emotional bias of the children only emerged in emoji faces, not in real faces. The above studies imply that emotional valence of faces can affect IB differently among different age groups. This study was designed to follow this line of research to further clarify the effect of emotional valence on IB, comparing children's and adults' emotional attentional bias in the same task. The results of the study may contribute to our understanding regarding the relationship between emotional valence of faces and IB of people of different ages.

Though there is no direct research investigating the agerelated difference of emotional bias based on IB, studies that have explored bias under attentional conditions provide clues in this area. It has been established that younger adults exhibit emotionally negative bias (Charles et al., 2001; Hilimire et al., 2014; Meng et al., 2015); however, conflicting results are reported for elderly adults' emotional bias. It has been demonstrated that when the faces are shown for long enough $(4,000 \mathrm{~ms})$, elderly adults showed a higher preference for emotionally positive faces than for negative faces (Leclerc \& Kensinger, 2010). Yet, an opposite result has been found when the faces are presented for a limited time $(200 \mathrm{~ms})$
(Wright et al., 2006). It seems that the presentation duration of the faces is an important factor affecting elderly adults' emotional bias under an attentional condition. It is noteworthy that the exposure duration of the picture has an effect on an adult's emotional bias to task-irrelevant faces ( $\mathrm{Yu}$ et al., 2015), which conveys the message that no matter what faces serve as attentional or unattentional stimuli, the duration of face presentation may affect elderly adults' attentional bias. Accordingly, it is significant to know whether the emotional bias would be found when the faces are shown for between $200 \mathrm{~ms}$ and 4,000 ms and attention is concentrated on another attentional task in elderly adults. This study investigated this age group's emotional bias under an inattentional condition, where the faces were presented unexpectedly for $1,000 \mathrm{~ms}$ while the elderly adults focus on another task. The ability to process emotional faces has adaptive value and is essential for successful social interactions and interpersonal relationships (Carstensen et al., 1998). In return, an individual's experience of social interactions and interpersonal relationships also exerts an influence on emotional bias along with personal cognitive factors (Ebner \& Johnson, 2009; English \& Carstensen, 2015). This suggests that there is age-related emotional bias with increasing age, especially among two groups of people with a large age difference (such as elderly adults vs. children).

Regarding children's emotional bias, research also shows that differences exist among children whose age and developmental characteristics are different. It has been confirmed that preschoolers have positive emotional bias under attentional conditions (Denham, 1986; Durand et al., 2007; Garner et al., 1994). However, it seems that typically developing school-aged children's emotional bias was not stable. A number of studies compared emotional bias between typically developing school children and children with special needs (above 7 years of age) who were autistic or had abuse experiences or anxiety characteristics (Pollak \& Tolley-Schell, 2003; Waters \& Lipp, 2008; Waters et al., 2010). The researchers found that the children with special needs demonstrated emotional bias to negative faces in attentional conditions, whilst their typically developing peers showed no significant emotional bias. However, typically developing children also showed negative emotional bias when the emotional faces were embedded among neutral faces or the scrambled pictures of a person that would distract children's attention (Chen et al., 2011; Waters et al., 2008). These results indicate that typically developing children's emotional bias is associated with the background of the facial pictures.

In addition to the emotional valence, the age of the faces could also affect people's face detection. It has been found that individuals have own-aged bias $(\mathrm{OAB})$ in face recognition, which means that people have the ability to recognize the faces of own-aged people better than the faces of other-aged individuals (Rhodes \& Anastasi, 2012). Previous studies 
reveal that people of any age group including from preschool children to older adults may exhibit a recognition bias to ownaged faces when the age span of facial material and age span of participants are perfectly matched as well as when the age span differs in each group (from 3 to 34 years) (Anastasi \& Rhodes, 2005; Wright \& Stroud, 2003). This study inspires the investigation of face-age bias that could be undertaken in a group with a certain age span. Relevant research also identifies the $\mathrm{OAB}$ occurrence's association with attentional resource. Neumann et al. (2015) found that OAB appeared in a face recognition and memorization task among younger and elderly adults whose attention was focused on this task or divided by a visual or an auditory interference task. However, OAB was weakened among younger adults when the divided attentional task occupied most of their attentional resources (Wang et al., 2017). These findings indicate that attention resource can modulate $\mathrm{OAB}$ in face recognition, yet little research has explored whether $\mathrm{OAB}$ exists when the participants' attention resource is fully directed to another task. This current study addresses this issue with the application of different ages of the faces presented in an IB task. IB is viewed to be an ecologically valid method for investigating attentional capture by unexpected occurrences (Lee \& Telch, 2008; New \& German, 2015), which is associated with the self-protection ability to perceive potentially urgent and lifethreatening information (Beanland et al., 2017).

To sum up, previous research provides robust evidence showing that age and emotional valence are associated with an individual's attentional bias, and this relationship is associated with the age of participants and the attention resource that includes attentional (expected stimuli), divided (semi-expected stimuli), and inattentional (unexpected stimuli) conditions. The research reveals characteristics of attentional process in humans across different age groups, although the majority of such research was undertaken under attentional conditions, and only focused on one age group in one set of experimental tasks. Little existing research compared the emotional attentional bias among different age groups, especially among school-aged children and middle-aged adults. In the present study, we focus on these two groups with a large age gap (school-aged children and mature middle-aged adults) and explore their attentional bias with a number of faces presented with different ages and emotional valence using an IB paradigm design. This study sought to address the following questions:

1) Do school-aged children and mature middle-aged adults show different IB with unexpected facial stimuli?

2) Does the emotional valence of faces effect the IB of school-aged children and mature middle-aged adults differently?

3) Does the age of the faces affect the IB of school-aged children and late middle-aged adults differently?

\section{Method}

\section{Participants}

Generally, the sample size of an experimental study should have statistical significance, which requires at least 15 participants in each experimental group (Zhu, 2011). In many IB studies, however, to ensure the reliability of results, researchers usually selected about 20 participants for each group (e.g., Devue et al., 2009; Mack et al., 2002; Zhang et al., 2016). In the light of these IB studies, we decided to recruit around 20 children and 20 adults for each experimental group.

Originally, 80 children (7- to 15 -year-olds) and 82 adults (40- to 70-year-olds) residing in Jiangxi, an inland province in China, participated in this study. Children were recruited from a local rural primary school and a middle school. Adults were recruited from residential districts nearby the schools. Participants were expected to meet the selection criteria including: (1) have normal or corrected-to-normal vision (all participants met this standard), (2) be unaware of the fundamental purpose of the study (all participants met this standard), (3) be able to identify a facial emotion in the full attentional trial (two children and three adults did not meet this standard). Finally, the data of 78 children $\left(M_{\text {age }}=10.62, S D\right.$ $=2.32 ; 29$ boys) and 79 adults $\left(M_{\mathrm{age}}=54.63, S D=8.192 ; 32\right.$ men) were analyzed. The age difference was controlled in the group of children, $\mathrm{F}(3,74)=0.273, p=0.844$, and the group of adults, $\mathrm{F}(3,75)=2.242, p=0.09$.

The ethics of this study were approved by the Ethics Committee of the Institute of Psychology, Zhejiang Normal University. Consent forms were collected from all the participants, who read the information letter and acknowledged the research topic and the data collection process.

\section{Materials}

The experiment was undertaken in a quiet and comfortable room with sufficient illumination. Participants sat in front of a computer monitor with a viewing distance of approximately $60 \mathrm{~cm}$ to complete the experimental tasks. The IB experiment design in this study was similar to the typical "cross-judging" task originated by Mack and Rock (1998) who used the software E-prime 3.0, and presented on a 17-in. Acer monitor with a resolution of $1,280 \times 768$ pixels and a refresh rate of $85 \mathrm{~Hz}$.

Sixteen photographs were taken of four child actors (age 715 years; two males, two females) and four adult actors (age 47-66 years; two males, two females) who were chosen for the experiment. Each actor performed two types of emotional expression (happy and angry). To ensure the age and emotional valence characteristic of the faces were sufficiently distinguishable, 20 college students conducted a consistent assessment of the age and emotional valence of the 16 faces. Four 
faces were chosen, with $90 \%$ of the students deeming them to be suitable for representing the unexpected stimuli for the study. The four faces included: adult-positive face (AP), adult-negative face (AN), child-positive face (CP), childnegative face $(\mathrm{CN})$. The four faces were printed in black and white and then framed within a gray oval, which assisted in the exclusion of relevant and distract factors, such as hairstyles and clothing. The photos were of the same size and brightness. Figure 1 displays the faces used in the experiment.

\section{Procedures}

The IB experiment design in this study is similar to the typical "cross-judging" task originated by Mack and Rock (1998). Considering the developmental differences between schoolaged children and mature middle-aged adults, we selected the classical static IB paradigm and made some modifications of the concrete parameters in the "cross-judging" task to make it less challenging than the typical "cross-judging" task in order to allow both the children and the adults to complete the task.

In the present study, we used realistic faces as critical stimuli, and these faces need more time and attention resource to process (Wang et al., 2012; Zheng, 2018), which is different from the icon-like faces and objects used in Mack and Rock's (1998) study. In the study of Devue et al. (2009), participants' (college students) recognition rate of realistic faces in a critical trial is $29 \%$ and the recognition rate in a full attentional trial is $50 \%$. This result indicates that the exposure time of $200 \mathrm{~ms}$ for faces is too short and it is difficult for participating college students to recognize the faces in a full attentional condition. In relevant studies, a participant-selection process usually includes full attentional trials. If participants cannot recognize the critical stimulus in full attentional trials, these participants would be identified as in-valued participants and they would be removed from the analysis (e.g., Murphy \& Greene, 2016). Given the recognition of unexpected faces was one of the judgment criteria of IB in the present study and the participants' (school-aged children and mature middle-aged adults with college education) cognitive abilities may be weaker than college students', the 200-ms exposure time for the target cross could be too challenging for our participants. If we still

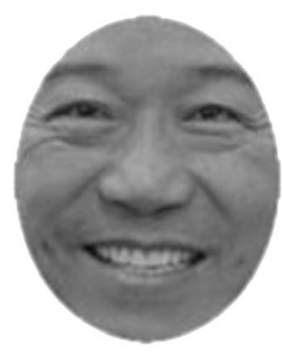

a

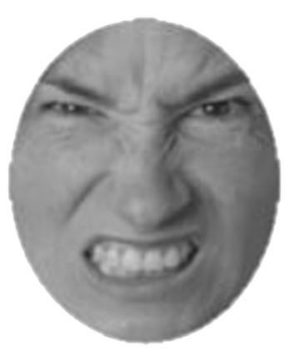

b applied the duration of $200 \mathrm{~ms}$, the IB rates would be high in both experimental groups, making the irrelevant variable (exposure time) a stronger factor in influencing IB than emotional valence and face age. Therefore, we used Wang and her colleagues' (in press) approach, which prolonged the exposure time for the target stimulus from $200 \mathrm{~ms}$ to $1,000 \mathrm{~ms}$ when exploring the emotional valence and facial type effects on preschooler's IB. This approach helps to reduce the negative influence of an inadequate exposure time on the IB task.

After the appearance of the target cross, a visual mask was presented for $500 \mathrm{~ms}$, which is similar to the study of Mack and Rock (1998). The purpose of this step was to eliminate the visual processing for the target cross after it disappeared (which is called a masking effect). Di Lollo et al. (2004) found that an important factor governing the strength of masking is the strength of the rapid transient response triggered by the onset of the mask, not its duration, and they found that the masking effect also appeared when the target duration outlasted the mask duration. Accordingly, we used the same mask duration (500 ms) as Mack and Rock's design.

Each trial of the IB task consisted of the following steps: (1) a tiny black central cue is displayed on the white screen for $1,000 \mathrm{~ms}$ to create a focus on the screen for the participants; (2) a target cross appears at the center of the screen for 1,000 $\mathrm{ms}$; (3) the cross is then covered by a visual mask with a black and gray pattern lasting for $500 \mathrm{~ms}$; (4) participants are asked to state which arm (horizontal or vertical) of the cross is longer. Considering the possibility that mature middle-aged adults and children may have individual differences in this cross-judgment task, we selected a larger size of cross than that of Mack and Rock's design to avoid the effect of the primary task's difficulty on IB. There are two combinations of the cross: 1,074 pixel $\left(9.2^{\circ}\right) \times 528$ pixel $\left(4.6^{\circ}\right)$ and 528 pixel $\left(4.6^{\circ}\right) \times 1,074$ pixel $\left(9.2^{\circ}\right)$. The difference between the vertical and horizontal lines is approximately $4.6^{\circ}$. The two sets of crosses appeared pseudo-randomly and evenly.

There were nine trials including three practice trials and six formal trials in the experiment. Before the practice trials, participants were informed to focus on the center of the screen and judge which arm of the crosses that appear is longer (see Fig. 2). The participants should respond with verbal language and the experimenter presses the corresponding keyboard
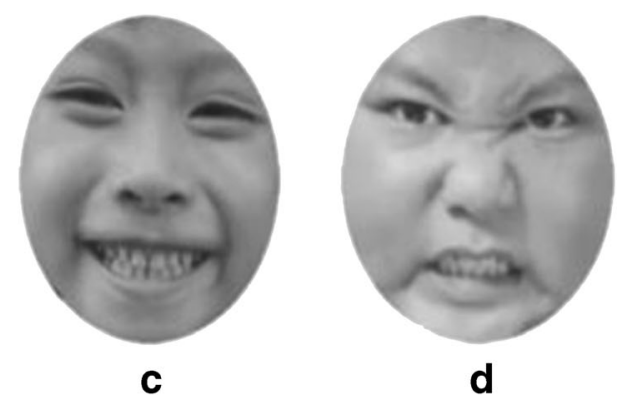

Fig. 1 Face stimuli used in the experiment 


\section{$1^{\text {st- } 33^{\text {rd }} \text { trials }}$}
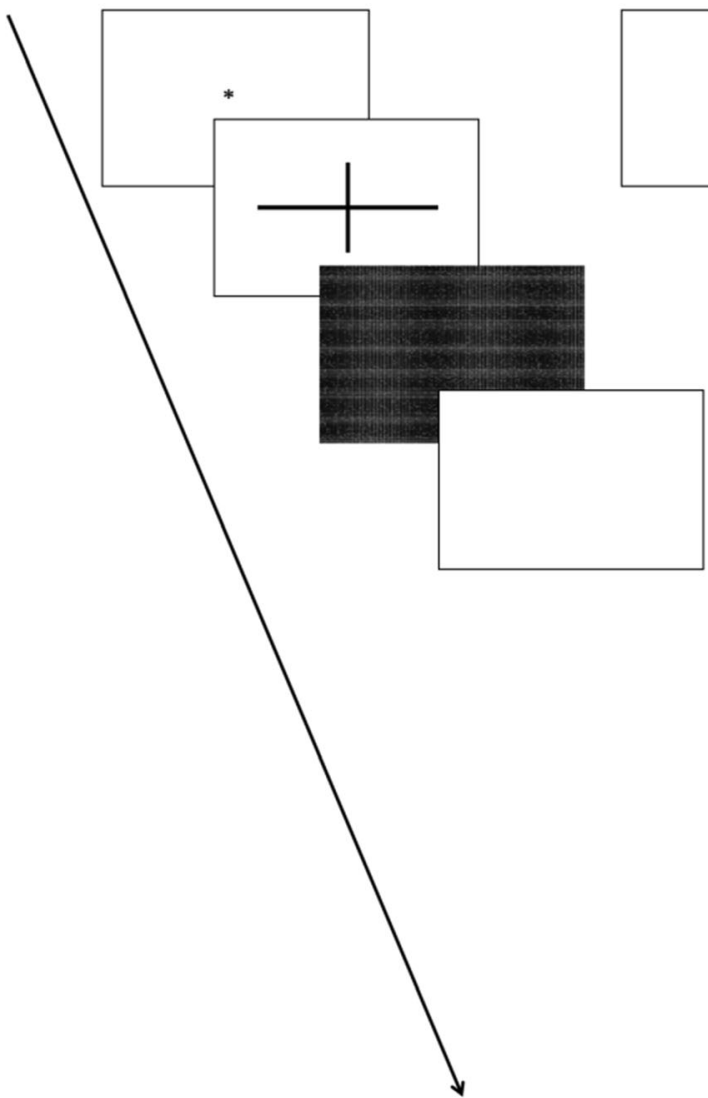

$4^{\text {th }}-6^{\text {th }}$ trials

$1000 \mathrm{~ms}$

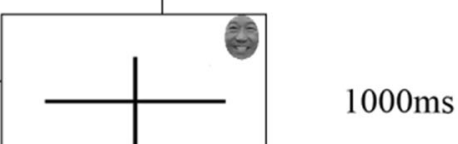

$500 \mathrm{~ms}$

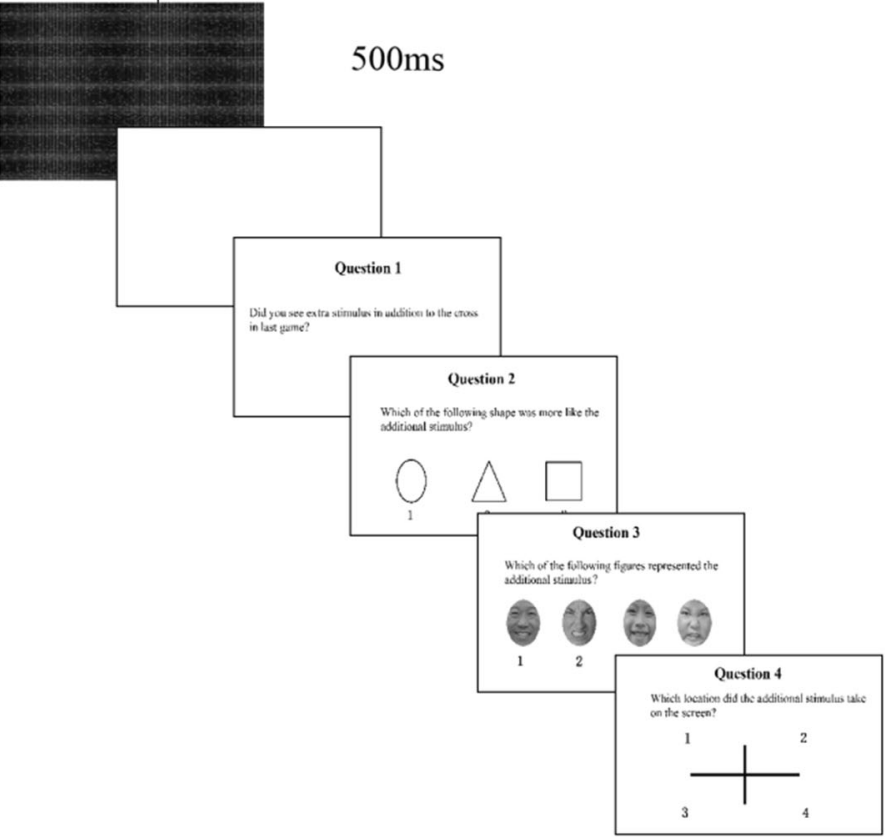

Fig. 2 Formal trials in the experiment

according to their response. Only when the participants fully understood the instructions and they completed the practice trials successfully, would the formal trials be undertaken. In our study, all the participants showed a good understanding of the instructions in these three trials.

The experiment included six formal trials; Fig. 2 shows the trials. The first three trials were general tests in which only the cross was presented. The purpose of these three trials was to familiarize the participants with the primary task of identifying the longer arm of the crosses. The fourth trial was critical, where the age group of the participants was divided into four sub-groups based on the four types of unexpected stimuli (face images of AP, AN, CP, and CN). The face would appear in the second quadrant of the cross shown on the screen. After completing the judgmental task, the participants were asked four questions that would indicate whether IB had occurred: Q1. "Did you see anything else on the screen?" Q2. "Which of the following shapes is most like the extra stimulus?" Q3. "Which of the following facial images could represent the extra stimulus?" Q4. "In which quadrant was the extra stimulus located on the cross?" Participants' answers were recorded by their experimenters.

As the questions provided the participants with a clue that a stimulus may appear during the tasks, the participant would then pay attention to any follow-up stimuli. Therefore, the next trial was conducted under a divided attentional condition and the last trial was a full attentional trial. Prior to the last trial, the experimenter told the participant to observe the extra stimulus rather than focusing on the cross, and that there was no need to judge the cross. This was a trial to exclude the participants who could not detect the facial image in the full attentional condition (see the selection criteria in the Participants section).

\section{Data coding}

Since the present study aimed to evaluate the participants' IB associated with the detection of different faces when they appeared unexpectedly, the results in the critical trial (the fourth formal trial) needed to be analyzed systematically to identify whether IB occurred among the participants under an inattentional condition that was created by the first four formal trails. In previous studies, researchers used a coding scheme that required participants to report that they were aware of unexpected stimuli and correctly selected one or two of the physical properties of the stimuli (Stothart et al., 2015), such as location, shape, or color. Considering the unexpected stimulus of the current study was an emotional face, 
which is different to the symbolic stimuli (geometric figures) in previous studies, we planned to carry out a further coding for the face recognition. If participants could select the shape (Q2) and the figure (Q3) of the unexpected stimulus correctly, or select the right location $(\mathrm{Q} 4)$ and the figure $(\mathrm{Q} 3)$ of the unexpected stimulus, the participant would be considered as a None-IB group (Wang et al., in press). Otherwise, they would be classified in the IB group.

All the experimental data were statistically analyzed with SPSS23.0, and binary logistic regression analyses were performed to examine the singular and interactive effects of the three independent variables on both the None-IB and IB groups: the age group of the participants, the emotional valence of the faces, and the age of the faces.

\section{Results}

\section{The accuracy of the primary task}

The mean accuracy of the primary task regarding the judgment of the cross' arms for adults was $99.49 \%$, and for children was $98.46 \%$. The $t$-test results revealed that there was no significant difference between children and adults in terms of the accuracy of the primary task, $t(155)=-1.471, p=0.143$.

\section{Detection rates of the unexpected stimuli}

The results of the detectors and detection rates of the four subgroups (AP, AN, CP, CN) among the children and the adults are displayed in Table 1.

A binary logistic regression method showed that there was a significant difference between the two age groups in terms of the detection rate, $\mathrm{B}=0.846$, Wals $=5.371, p<0.05,95 \% \mathrm{CI}$ $[1.139,4.766]$. Children detected the unexpected stimuli better than adults (37.18\% vs. $20.25 \%)$. However, the effect of the age of the faces was not a significant factor for predicting the detection rate in the two groups, $\mathrm{B}=0.134$, Wals $=0.143$, $p>0.05,95 \%$ CI $[0.572,2.285]$. Emotional valence of the

Table 1 The number of detectors and detection rates of the different experimental groups

\begin{tabular}{lllllllll}
\hline \multirow{2}{*}{ Group } & \multicolumn{3}{l}{ Child } & \multicolumn{3}{l}{ Adult } \\
\cline { 2 - 5 } \cline { 7 - 8 } & IB & NIB & Detection rate & & IB & NIB & Detection rate \\
\hline Adult-positive face & 14 & 5 & $26.32 \%$ & & 13 & 6 & $31.58 \%$ \\
Adult-negative face & 12 & 8 & $40 \%$ & & 17 & 2 & $10.53 \%$ \\
Child-positive face & 12 & 8 & $40 \%$ & & 15 & 6 & $28.57 \%$ \\
Child-negative face & 12 & 8 & $42.11 \%$ & & 18 & 2 & $10 \%$ \\
Total & 49 & 29 & $37.18 \%$ & & 63 & 16 & $20.25 \%$ \\
\hline
\end{tabular}

faces was also not a significant element influencing the two groups' detection rates, $\mathrm{B}=-0.295$, Wals $=0.690, p>0.05$, $95 \%$ CI $[0.372,1.492]$. These findings indicate that children's detection ability of the unexpected stimuli of the faces is better than that of the adults in general, regardless of the type of face.

A significant interaction effect was observed between the age groups of the participants and emotional valence of the faces (see Fig. 3), $\mathrm{B}=1.652$, Wals $=4.409, p<0.05,95 \% \mathrm{CI}$ $[1.116,24.386]$. The simple effect of the emotional valence is obvious, $\mathrm{B}=-2.974$, Wals $=4.881, p<0.05,95 \%$ CI $[0.004$, $0.715]$. To further clarify this interaction effect, we analyzed the simple effect of emotional valence on the children and the adults separately. For children, the main effect of emotional valence was not significant, $\mathrm{B}=0.330$, Wals $=0.493, p>$ $0.05,95 \%$ CI $[0.553,3.499]$. For adults, the main effect of emotional valence was significant, $\mathrm{B}=-1.322$, Wals $=4.394$, $p<0.05,95 \%$ CI $[0.077,0.918]$. Adults were significantly more likely to detect the positive face than the negative face $(41.03 \%$ vs. $10.26 \%)$. There was no interaction effect between the age groups and the age of the faces, $\mathrm{B}=0.426$, Wals $=$ $0.338, p>0.05,95 \%$ CI $[0.365,6.418]$. No effect was found in an Emotional Valence of Faces $\times$ Age of Faces interaction, $\mathrm{B}=-0.241$, Wals $=0.115, p>0.05,95 \%$ CI $[0.195,3.165]$. The findings demonstrate that among the four types of facial stimuli, both AP and CP types would be more likely to capture the adults' attention under an inattentional condition, although the adults had a lower detection rate than the children.

\section{Discussion}

This study used an IB task to examine the effect of facial images with different emotional valence and age on children and adults' IB. Given the fact that little research has been undertaken in the exploration of age-related effects on emotional and age bias of facial images under an inattentional condition, this study is necessary and significant because it increases our knowledge about how different types of facial image may impact children's and adults' attentional process when they focus on another task. The findings reveal the different characteristics of children's and adults' attentional bias on the same facial stimuli, which enhances our understanding about human's attentional processes in different life stages.

An important finding from the study is that children have a higher detection rate of unexpected faces than adults, which indicates that children's attention could be more easily captured when they are concentrating on a specific project. This finding is in accordance with previous research that shows age-related variations in IB and a decline in detection rates for unexpected stimuli from childhood to adulthood (Horwood \& Beanland, 2016; Liu, 2018; Stothart et al., 2015). Furthermore, this study confirms the notion that children's involuntary attention predominates in their daily life 


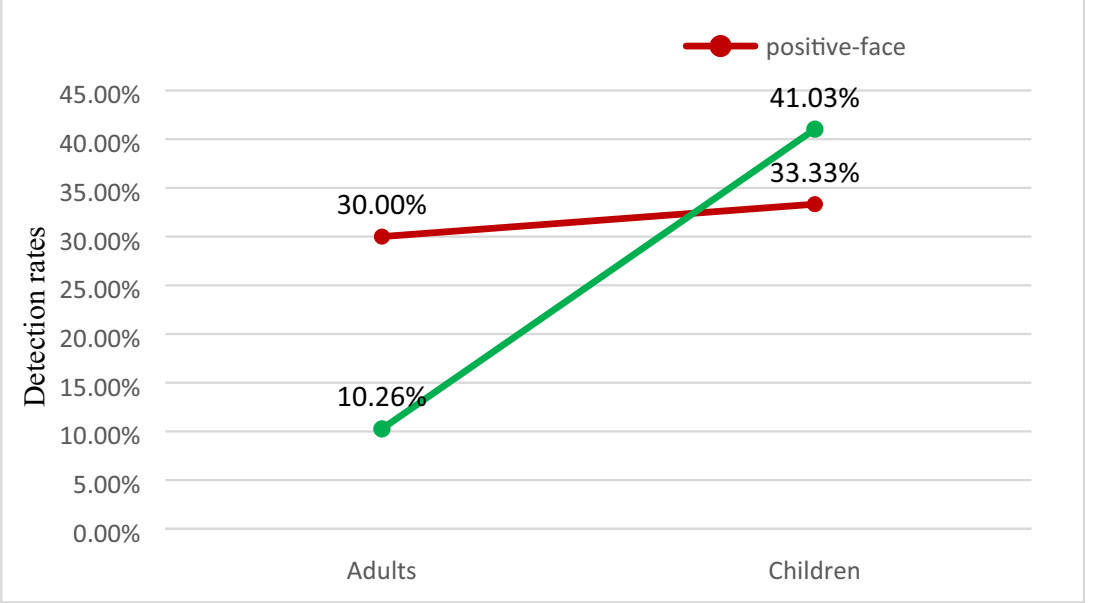

Fig. 3 The interaction between the age of participants and emotional valence

(Gomes et al., 2000; Wetzel et al., 2006). This has an educational implication for the environmental set-up for children in that an environment with less unexpected stimuli is needed to facilitate children's focus on a project. This characteristic is very different from adults who seem to have the ability to focus on a task in a distracting environment by ignoring unexpected stimuli. This is an important attribute of an adult's attentional process.

The results regarding the effect of emotional valence on the children and the adults' IB deserve further consideration. In our study, only adults present positive emotional bias, children do not show any. This is consistent with several previous studies' that showed that elderly adults are more sensitive to emotionally positive faces under attentional conditions (Hilimire et al., 2014; Leclerc \& Kensinger, 2010; Meng et al., 2015), a phenomenon termed a "positive effect" of elderly adults. The mechanism of this positive effect is diversified in the literature (Liu, 2018). As mentioned before, the exposure time may be a key factor for the attentional bias of faces. Negative attentional bias appears in a relatively earlier time (Wright et al., 2006) and positive attentional bias appears relatively late (Isaacowitz et al., 2018). In this study, the exposure time of the facial image is $1,000 \mathrm{~ms}$, which is relatively long, and we found a positive attentional bias in mature adults. Furthermore, according to the "social emotional selectivity theory" focusing on social and development factors (English \& Carstensen, 2015), a positive effect in an IB study may be explained by the fact that mature middle-aged people have many social experiences and thus may have more understanding in the power of positive emotions. Research shows that mature adults tend to have a desire to develop positive emotions for gaining optimistic social experiences (Lang, 2000), and they are more liable to take a positive and compliant attitude when facing heavy stress or emotional conflicts (Charles et al., 2001). People who have a positive emotional state over a long time are more sensitive to the perception of positive emotions (Diener \& Diener, 1996). The adult participants of this study were mature middle-aged people $(54.63 \pm$ 8.192 years old) who lived in rural inland China. They might have had extensive life experiences and may have undergone many stressful situations. The adult participants' backgrounds might predict that they would be sensitive to the positiveemotional face. This study confirms the "positive effect" for mature middle-aged adults' emotional bias that is similar to that of elderly adults from a perspective of IB.

The finding that children had no attentional bias with regard to an emotional face is in accordance with previous studies that found school-aged children showed no attentional bias even when emotional faces occurred expectedly (Pollak \& Tolley-Schell, 2003; Waters et al., 2008; Waters et al., 2010). Studies consistently indicate that school-aged children's attentional bias for emotional faces may not be as obvious as that of adults in any attention condition (Ge \& Jing, 2015; Hilimire et al., 2014; Leclerc \& Kensinger, 2010; Meng et al., 2015). Nevertheless, an IB study on preschool children came to the inconsistent result that young children's IB could be affected by the presentation styles of the faces. They did not present attentional bias for the emotional real-person faces that is very similar to the photos used in the current study. However, when the face was presented in the style of an emoji, where the facial expression was exaggerated to the utmost extent, an effect of the emotional valence on these preschool children's IB occurred (Wang et al., in press). Wang et al. (in press) indicate that emoji-styled faces can attract preschool children's attention more easily than real-person's photos, which offers a new avenue for research relating to the effect of emotional valence on people's IB. However, according to Wang's study, a question can be raised regarding the result of the current study and whether the children would perform differently if the emotional faces were presented in an emoji style in the IB task to the school-aged children. 
Further study is needed to explore the effect of emojistyled face on school-aged children's emotional bias.

In addition, the results of having no significant effect of face age on both adults and children's IB could be attributed to the divided attentional resources and interference of emotional valence in the research design. Previous studies demonstrated that $\mathrm{OAB}$ is regulated by attention resources (Wang et al., 2017). Only when the attentional resources are sufficient in a focused attentional condition (Bernstein et al., 2007; Rhodes \& Anastasi, 2012) or a divided attentional condition where the intensity of attention distribution is weak (Neumann et al., 2015) may people perform OAB. Moreover, the emotional valence of faces may also influence people's $\mathrm{OAB}$ (Ebner \& Johnson, 2009; Ebner et al., 2013). Ebner et al. (2013) used emotional faces to explore whether OAB of young and old adults would be affected by the faces' emotions at different ages and found that $\mathrm{OAB}$ disappeared under such conditions. The present study reinforces the idea that the emotion of a face is more influential than the age of the face.

A noteworthy point is the high accuracy of the primary task (around 99\%) in this study, which is much higher than the accuracy result (around 70\%) in some other related studies (Devue et al., 2009; Mack \& Rock, 1998). Comparably high accuracy rates were found in recent IB studies focusing on preschoolers (around 98\%) and primary school children with autism spectrum disorder (around 97\%) (Murphy \& Greene, 2016; Swettenham et al., 2014). These studies show that children can perform well in a cross-judgment task. The excellent performance in the primary task in this study reflects that participants were fully engaged in the primary task. However, there is another possibility that the high accuracy of the primary task may relate to the primary task being too easy for these participants. It is hard to verify this assumption in this study, but we think that the low detection rates of unexpected stimuli may imply that the easy primary task led the participants to detect the unexpected stimuli less effectively. In other words, whether the primary task is too easy here seems not be an important factor in the attentional bias in this study.

Last but not least, we should mention that the presentation time of the unexpected stimuli in the current study was 1,000 $\mathrm{ms}$, which is different to Mack and Rock's 250-ms time. This design may result in the mechanism of IB in Mack and Rock's study being different to that in the current study (the reason why we increased the presentation time is explained in the Procedure section). Basically, limited resources may be the most important mechanism of IB in Mack and Rock's study in which the cross was presented only for $250 \mathrm{~ms}$ (Lavie \& Tsal, 1994). However, IB would not only occur when the attentional resources are limited and the attentional set to targeted (expected) stimuli could also affect participants' inattention to the untargeted stimuli even when the presentation time is longer, such as $500 \mathrm{~ms}$ (Eitam et al., 2013) or $5 \mathrm{~s}$ (Most et al., 2005). Hence, the IB rate in the current study is high (nearly $80 \%$ ), and we think that most of the participants were still inattentional when the unexpected stimuli was presented for $1,000 \mathrm{~ms}$.

\section{Limitations and future research}

This study contributes to the field of attentional bias by identifying the effect of emotional valence and age of faces on IB of school-aged children and mature middle-aged adults with strong evidence; however, a few limitations exist in the participant selection and the research design. Firstly, the age span between the children's and the adult's groups is considerable. The 79 adults' ages varied from late 40s to early 60 s (54.63 \pm 8.192 ), and the 78 children's ages were from eight to almost 13 years $(10.62 \pm 2.32)$. Although there were no consistent dividing criteria for school-aged children and middle-aged adults (Anastasi \& Rhodes, 2005; Liu, 2018), and in the previous IB studies relating emotional attentional bias and $\mathrm{OAB}$, the age-related effect was discovered in groups with large age spans. The results of the study would be more reliable if the age span was more contracted. Secondly, the emotional valence didn't include a neutral emotional face. As mentioned before, emotional valence is an important factor moderating the $\mathrm{OAB}$ effect. If a neutral emotional face (emotionless face) was included in the IB task of the study, the effect of the face's age on the participants' IB could be different from that of emotional faces. Thirdly, real faces in our daily life always occur in a dynamic condition, thus a sustained IB task should be applied in future studies, which could increase the ecological validity. Fourthly, the data collection relied on the participants' verbal responses to the designed questions. If the eyelink technical method was applied to support the data collection, the reliability of the study would be increased. Finally, the angry and happy faces differ in arousal level, which would affect individual's attentional bias. When selecting the face material, the important factor of arousal of the stimuli needs to be taken into account in future studies. Future research addressing the limitations would extend our understanding about human being's attentional bias, and capture it in different life stages with more robust evidence.

Author Note This research was supported by a grant from Zhejiang Natural Science Foundation (The effect of threat stimulus types on children's inattentional blindness: from behavior to neural mechanism, LQ20C090003).

\section{References}

Anastasi, J. S., \& Rhodes, M. G. (2005). An own-age bias in face recognition for children and older adults. Psychonomic Bulletin \& Review, 12(6), 1043-1047. https://doi.org/10.3758/BF03206441. 
Beanland, V., Jeans, R., Filtness, A. J. \& Jeans. R. (2017). Change detection in urban and rural driving scenes: effects of target type and safety relevance on change blindness. Accident Analysis and Prevention.100, https://doi.org/10.1016/j.aap.2017.01.011.

Bernstein, M. J., Young, S. G., \& Hugenberg, K. (2007). The crosscategory effect: mere social categorization is sufficient to elicit an own-group bias in face recognition. Psychological Science, 18(8), 706-712. https://doi.org/10.1111/j.1467-9280.2007.01964.x.

Carstensen, L. L., Gross, J. J., \& Fung, H. (1998). The social context of emotional experience. In K. W. Schaie \& M. P. Lawton (Eds.), Annual review of gerontology and geriatrics (Vol. 17, pp. 325352). New York: Springer.

Charles, T. T., Reynold, C. A., \& Gatz, M. (2001). Age-related differences and change in positive and negative affect over 23 years. Journal of Personality and Social Psychology, 80, 136-151. https://doi.org/10.1037/0022-3514.80.1.136.

Chen, S. S., Bai, X. J., Shen, D. L., Yan, G. L., \& Zhang, L. C. (2011). Emotional Faces Detection and Processing of Individuals with Autism Spectrum Disorder Aged 7-10. Psychological Development and Education, 5, 449-458. https://doi.org/10.16187/ j.cnki.issn1001-4918.

Denham, S. A. (1986). Social cognition, prosocial behavior, and emotion in preschoolers: contextual validation. Child Development, 57(1), 194-201. https://doi.org/10.1111/j.1467-8624.1986.tb00020.x.

Devue, C., Laloyaux, C., Feyers, D., Theeuwes, J., \& Brédart, S. (2009). Do pictures of faces, and which ones, capture attention in the inattentional-blindness paradigm? Perception, 38(4), 552-568. https://doi.org/10.1068/p6049.

Di Lollo, V., Mühlenen, A. V., Enns, J. T., \& Bridgeman, B. (2004). Decoupling stimulus duration from brightness in metacontrast masking: data and models. Journal of Experimental Psychology Human Perception \& Performance, 30(4), 733-745. https://doi. org/10.1037/0096-1523.30.4.733

Diener, E., \& Diener, C. (1996). Most people are happy. Psychological Science, 7(3), 181-185. https://doi.org/10.1111/j.1467-9280.1996. tb00354.x.

Durand, K., Gallay, M., Seigneuric, A., Robichon, F., \& Baudouin, J. Y. (2007). The development of facial emotion recognition: the role of configural information. Journal of Experimental Child Psychology, 97(1), 14-27. https://doi.org/10.1016/j.jecp.2006.12.001.

Eastwood, J. D., Smilek, D., \& Merikle, P. M. (2003). Negative facial expression captures attention and disrupts performance. Perception \& Psychophysics, 65(3), 352-358. https://doi.org/10.3758/ BF03194566.

Ebner, N. C., \& Johnson, M. K. (2009). Young and older emotional faces: are there age-group differences in expression identification and memory. Emotion, 9(3), 329-39. https://doi.org/10.1037/a0015179.

Ebner, N. C., Johnson, M. R., Rieckmann, A., Durbin, K. A., Johnson, M. K., \& Fischer, H. (2013). Processing own-age vs. other-age faces: neuro-behavioral correlates and effects of emotion. NeuroImage, 78 , 363-371. https://doi.org/10.1016/j.neuroimage.2013.04.029.

Eitam, B., Yeshurun, Y., \& Hassan, K. (2013). Blinded by irrelevance: Pure irrelevance induced "blindness". Journal of Experimental Psychology: Human Perception and Performance, 39(3), 611-615. https://doi.org/10.1037/a0032269.

English, T., \& Carstensen, L. L. (2015). Does positivity operate when the stakes are high? health status and decision making among older adults. Psychology \& Aging, 30(2), 348-355. https://doi.org/10. 1037/a0039121.

Garner, P. W., Jones, D. C., \& Miner, J. L. (1994). Social competence among low-income preschoolers: emotion socialization practices and social cognitive correlates. Child Development, 65(2), 622637. https://doi.org/10.1111/j.1467-8624.1994.tb00772.x.

Ge, G. H., \& Jing, Y. B. (2015). The effect of ecological significance to unexpected stimulion inattentional blindness: the role of emotional valence and fine degree. Psychological Development and
Education, 31(6), 661-667. https://doi.org/10.16187/j.cnki. issn1001-4918.

Gomes, H., Molholm, S., Ritter, W., Kurtzberg, D., Cowan, N., \& Vaughan-JR, H. G. (2000). Mismatch negativity in children and adults, and effects of an attended task. Psychophysiol, 37, 807816. https://doi.org/10.1017/S0048577200990632.

Hilimire, M. R., Mienaltowski, A., Blanchard-Fields, F., \& Corballis, P. M. (2014). Age-related differences in event-related potentials for early visual processing of emotional faces. Social Cognitive and Affective Neuroscience, 9(7), 969-976. https://doi.org/10.1093/ scan/nst071.

Horwood, S., \& Beanland, V. (2016). Inattentional blindness in older adults: effects of attentional set and to-be-ignored distractors. Attention Perception \& Psychophysics, 78(3), 818-828. https://doi. org/10.3758/s13414-015-1057-4.

Huang, S. S., Guo, Y. N., \& Zheng, X. F. (2012). The Effects of Unexpected Stimulus's Meaningfulness and Position to Inattentional Blindness in College Students. Psychological Development and Education, 2, 36-41. https://doi.org/10.16187/j. cnki.issn1001-4918.2012.02.012.

Isaacowitz, D. M., Livingstone, K. M., Richard, M., \& SeifEl-Nasr, M. (2018). Aging and attention to self-selected emotional content: A novel application of mobile eye tracking to the study of emotion regulation in adulthood and old age. Psychology and Aging, 33(2), 361-372. https://doi.org/10.1037/pag0000231.

Lang, F. R. (2000). Endings and continuity of social relationships: maximizing intrinsic benefits within personal networks when feeling near to death. Journal of Social \& Personal Relationships, 17(2), 155-182. https://doi.org/10.1177/0265407500172001.

Lavie, N., \& Tsal, Y. (1994). Perceptual load as a major determinant of the locus of selection in visual attention. Attention, Perception \& Psychophysics, 56(2), 183-197. https://doi.org/10.3758/ BF03213897.

Leclerc, C. M., \& Kensinger, E. A. (2010). Age-related valence-based reversal in recruitment of medial prefrontal cortex on a visual search task. Social Neuroscience, 5(5-6), 560-576. https://doi.org/10.1080/ 17470910903512296.

Lee, H. J., \& Telch, M. J. (2008). Attentional biases in social anxiety: an investigation using the inattentional blindness paradigm. Behaviour Research \& Therapy, 46(7), 819-835. https://doi.org/10.1016/j.brat. 2008.04.001.

Liu, H. H. (2018). Age-Related Effects of Stimulus Type and Congruency on Inattentional Blindness. Frontiers in Psychology, 9, 794. https://doi.org/10.3389/fpsyg.2018.00794.

Mack, A., Pappas, Z., Silverman, M., \& Gay, R. (2002). What we see: Inattention and the capture of attention by meaning. Consciousness \& Cognition, 11(4), 488-506. https://doi.org/10.1016/S10538100(02)00028-4Get.

Mack, A., \& Rock, I. (1998). Inattentional blindness. Cambridge, MA: MIT Press.

Meng, X., Yang, J., Cai, A., Ding, X., Liu, W., Li, H., \& Yuan, J. J. (2015). The neural mechanismsunderlying the aging-related enhancement of positive effects: electrophy siological evidences. Frontiers in Aging Neuroscience, 7, 143. https://doi.org/10.3389/ fnagi.2015.00143.

Most, S. B., Scholl, B. J., Clifford, E. R., \& Simons, D. J. (2005). What you see is what you set: Sustained inattentional blindness and the capture of awareness. Psychological Review, 112(1), 217-242. https://doi.org/10.1037/0033-295X.112.1.217.

Murphy, G., \& Greene, C. M. (2016). Perceptual load induces inattentional blindness in drivers. Applied Cognitive Psychology, 30(3), 479-483. https://doi.org/10.1002/acp.3216.

Neumann, M. F., End, A., Luttmann, S., Schweinberger, S. R., \& Wiese, H. (2015). The own-age bias in face memory is unrelated to differences in attention-evidence from event-related potentials. 
Cognitive Affective \& Behavioral Neuroscience, 15(1), 180-194. https://doi.org/10.3758/s13415-014-0306-7.

New, J. J., \& German, T. C. (2015). Spiders at the cocktail party: an ancestral threat that surmounts inattentional blindness. Evolution and Human Behavior, 36(3), 165-173.https://doi.org/10.1016/j. evolhumbehav.2014.08.004.

Pollak, S. D., \& Tolley-Schell, S. A. (2003). Selective attention to facial emotion in physically abused children. Journal of Abnormal Psychology, 112 (3), 323-338. https://doi.org/10.1037/0021-843x. 112.3.323.

Rhodes, M. G., \& Anastasi, J. S. (2012). The own-age bias in face recognition: a meta-analytic and theoretical review. Psychological Bulletin, 138(1), 146-174. https://doi.org/10.1080/13506285.2013. 823140.

Stothart, C. R., Boot, W. R., \& Simons, D. J. (2015). Using mechanical Turk to assess the effects of age and spatial proximity on inattentional blindness. Collabra, 1(1), 1-7. https://doi.org/10. $1525 /$ collabra.26.

Swettenham, J., Remington, A., Murphy, P., Feuerstein, M., Grim, K., \& Lavie, N. (2014). Seeing the unseen: autism involves reduced susceptibility to inattentional blindness. Neuropsychology, 28(4), 563570.https://doi.org/10.1037/neu0000042.

Wang, J. L., Zhang, H., \& Yang, M. (in press). The Effect of Emotional Valence and Facial Type on Children's Inattentional Blindness. Psychological Development and Education.

Wang, L, Wang, J. M., Wang, J. L., \& Lu, Y. J. (2012). A Comparative Event-related Potential Study on Recognition of Cartoon Face and Real Face. Psychological Research, 5(5), 19-28.

Wang, Z., Guo, D., Fang, W., \& Sun, Y. H. (2017). Attention modulates the own-age bias in face recognition. Chinese Science Bulletin, 62(15), 1620-1630. https://doi.org/10.1360/N972016-01323.

Waters, A. M., Kokkoris, L. L., Mogg, K., Bradley, B. P., \& Pine, D. S. (2010). The time course of attentional bias for emotional faces in anxious children. Cognition \& Emotion, 24(7), 1173-1181. https:// doi.org/10.1080/02699930903274355.

Waters, A. M., \& Lipp, O. V. (2008). Visual search for emotional faces in children. Cognition and Emotion, 22(7), 1306-1326. https://doi.org/ 10.1080/02699930701755530.
Waters, A. M., Mogg, K., Bradley, B. P., \& Pine, D. S. (2008). Attentional bias for emotional faces in children with generalized anxiety disorder. Journal of the American Academy of Child \& Adolescent Psychiatry, 47(4), 435-442. https://doi.org/10.1097/ CHI.0b013e3181642992.

Wetzel, N., Widmann, A., Berti, S., \& SchroGer, E. (2006). The development of involuntary and voluntary attention from childhood to adulthood: a combined behavioral and event-related potential study. Clinical Neurophysiology, 117(10), 2191-2203. https://doi.org/10. 1016/j.clinph.2006.06.717.

Wright, C. I., Wedig, M. M., Williams, D., Rauch, S. L., \& Albert, M. S. (2006). Novel fearful faces activate the amygdala in healthy young and elderly adults. Neurobiology of Aging, 27(2), 361-374. https:// doi.org/10.1016/j.neurobiolaging.2005.01.014.

Wright, D. B., \& Stroud, J. N. (2003). Age differences in lineup identification accuracy: people are better with their own age. Law and Human Behavior, 26(6), 641-654. https://doi.org/10.1023/A: 1020981501383.

Yu, J., Ma, Z. L., Niu, Y. N., Zhang, B. S., Broster, L. S., \& Li, J. (2015). The time course of age-related emotional preference in taskirrelevant affective processing. Progress in Biochemistry \& Biophysics, 42(4), 365-374. https://doi.org/10.16476/j.pibb.2014. 0372.

Zhang, H., Zhang, X., He, Y., \& Shi, J. (2016). Inattentional blindness in 9- to 10-year-old intellectually gifted children. Gifted Child Quarterly, 60(4), 287-295.https://doi.org/10.1177/ 0016986216657158

Zheng, C. Y. (2018). A comparative behavioral and event-related potentials studies on prechoolers' expression cognition of cartoon face and real face [Unpublished Master thesis]. Zhejiang Normal University.

Zhu, Y. (2011). Foundations of Experimental Psychology. Beijing: Peking University Press.

Publisher's note Springer Nature remains neutral with regard to jurisdictional claims in published maps and institutional affiliations. 